\title{
Adding an Interactive Display to a Public Basketball Hoop can Motivate Players and Foster Community
}

\author{
Alan Chatham, Florian 'Floyd' Mueller \\ Exertion Games Lab \\ RMIT University \\ Melbourne, Australia \\ \{alan, floyd\}@exertiongameslab.org
}

\begin{abstract}
Interactive displays that aim to engage people through play have been successfully deployed in urban environments. However, there has been little work bringing interactive displays into existing public game spaces like outdoor basketball courts. To explore this, we designed an interactive display for a public half-court basketball hoop. We studied the impact of 3 different display modes over a 10 -week period through interviews with players, spectators, and passers-by. Our findings suggest 3 dimensions for the design space of such interactive displays: balancing noticeability across different user groups, support for different play action, and support for connecting user groups. We also present 6 design tactics along these dimensions to help designers create engaging interactive displays for public game spaces.
\end{abstract}

\section{Author Keywords}

Public display, basketball, urban screens, public game space

\section{ACM Classification Keywords}

H5.m. Information interfaces and presentation (e.g., HCI): Miscellaneous.

\section{INTRODUCTION}

As display technology has improved and costs have dropped, display-based interactive experiences have become increasingly common, in particular in public spaces in urban environments. There are interactive broadcasts of sports events on large screens in public squares [7], games being played on projected displays on floors in shopping malls [8], interactive play experiences on public transport [5][32], etc., suggesting that designers and researchers are increasingly exploring the use of interactive displays in public spaces.

Many of these approaches to interactive displays in public spaces use digital games to introduce play into these spaces, intending to add a more playful dimension to urban life. In contrast, we point out that there are already many public spaces where people come together and play games, such as

Permission to make digital or hard copies of all or part of this work for personal or classroom use is granted without fee provided that copies are not made or distributed for profit or commercial advantage and that copies bear this notice and the full citation on the first page. Copyrights for components of this work owned by others than ACM must be honored. Abstracting with credit is permitted. To copy otherwise, or republish, to post on servers or to redistribute to lists, requires prior specific permission and/or a fee. Request permissions from permissions@acm.org.

UbiComp'13, September 8-12, 2013, Zurich, Switzerland.

Copyright (C) 2013 ACM 978-1-4503-1770-2/13/09 ..\$15.00

http://dx.doi.org/10.1145/2493432.2493518 public playgrounds and parks. In particular, sports fields such as basketball courts, soccer pitches, and baseball diamonds offer play experiences that can enhance urban life by providing opportunities for exercise and socialization while helping build a sense of community [6].

Our research focuses on interactive displays in these public game spaces in urban settings. We define public game spaces as publicly accessible spaces intended for play. These urban public game spaces can be further characterized as primarily outdoor environments where people come together to play sports without an administering organization. In particular, we see these public game spaces as a location for physical games offering opportunities for spectatorship, public participation, and fostering community.

We find these public game spaces to be distinct from both private game spaces for physical activity as well as professional sports arenas. In private game spaces, such as corporate sports facilities or indoor gyms, there are few opportunities for spectatorship, and the private nature of the space limits the potential for community socialization. Professional sports arenas, on the other hand, offer spectators opportunities to socialize with each other, but they offer little opportunity for public exercise. Additionally, participation in these locations is usually limited to professional athletes and games are played within the strict rules of a governing body.

Our research explores what happens when an interactive display is introduced to a public game space. We designed an interactive display that we installed on a publicly accessible outdoor half-court basketball court in a busy inner-city location. The display showed simple textual information based on a sensor that detected when a ball went through the hoop. The system operated 24 hours a day for a period of 10 weeks.

We experimented with 3 different display modes: a counter that displayed the total number of shots made during an entire day, a timer that displayed the number of seconds since the last basket was scored, and a mode in which a carefully crafted "trash talk" text was displayed when a basket was made. Through our design process, interviews with 164 players, audience members, and passers-by, we derived a set of design dimensions that characterize the 
design space of these displays. Our results also suggest how interactive displays can drive increased exercise motivation as well as foster an increased sense of community between the different users of the space. Lastly, we outline a number of strategies for the design of future interactive displays in public game spaces.

Our contribution is the first understanding of the impact of an interactive display in a public game space. In particular, we offer knowledge about the key design dimensions of interactive displays in public game spaces, interesting for researchers and designers who investigate interactions between displays and users. We also provide practical design advice for urban designers who want to support public game spaces with interactive technology. Moreover, our design suggests that such interactive displays need not be expensive or complex and can therefore readily be implemented in today's public game spaces.

\section{RELATED WORKS}

Several related works informed our research. In particular, we draw on prior research that investigated how people interact with public displays in urban environments offering digital game experiences. For example, O'Hara and Glancy examined how people interacted with displays in an innercity location that displayed a digital game for people to play in the space [22]. The authors found that interactions with public screen games are strongly influenced by the physical characteristics of the space, similar to the notion of considering the space in exertion games [20], reminding us to consider the layout of the basketball court and basket hoop. Furthermore, the authors found that public display games offer a performative social aspect not prevalent in most other digital games. This performative aspect is seen in non-game experiences as well, such as Peltonen et al.'s work with CityWall [26], and while we have seen projects which visualize existing behavior in a space, such as Paulos and Jenkins' Jetsam rubbish bin [25], we do not yet know how displays driven by existing play in public can change the relationships between participants and non-participants.

We also learn from Brignull and Rogers' work about the "Honey Pot Effect", where interaction with a screen can drive social clustering and further engagement [3]. This group interaction behavior helps us understand how users come to understand displays in public settings, however, there has been no work yet how this effect unfolds in public game spaces.

Research on ambient interactive displays has also informed our work, since users of public game spaces might have other goals as part of their urban life that makes interacting with a display the non-primary activity. Pousman and Stasko identify four design dimensions for ambient displays: information capacity, notification level, representation fidelity, and aesthetic concerns [28]. We find the notion of notification level to be particularly important; users in a public play space are there primarily to play, so a display must provide information without disrupting their play activity. We therefore explicitly examine how notifications unfold in a public game space context, in particular amongst its many different users.

Matthews et al. focused on peripheral displays and developed a framework for their development and evaluation based on activity theory [17]. The authors articulated a set of themes; in particular, we found the notions of awareness and distraction created by a display relevant as users of a public game space might not expect a display, and hence might have difficulty becoming aware of it. Furthermore, activity in a public game space can be very intense and physical, and any unwelcomed distraction could lead to disengagement or, even worse, physical injury. We extend this work by looking into how these themes unfold if there are multiple users in such a space, possibly having different needs and motivations.

Many projects have used peripheral displays in a multi-user workplace setting. For example, O'Hara et al. presented a system for room reservations in an office setting that uses peripheral displays. The authors examined how the displays affect people's relationships with the room [23]. Sturm et al. presented a peripheral display that detects signs of listener interest in a meeting setting and displays this information for all to see on a tabletop [31]. These systems demonstrate how peripheral displays can influence social behavior without being the main focus of attention. Our project extends these projects by moving into the non-work space of a public basketball court.

Other works have investigated augmenting the spectator experience in sporting arenas. For instance, the eStadium at Purdue as well as Bentley and Groble's work both aim to augment the spectator experience though multimedia presented via a mobile phone [1][2]. Pettula et al. investigated how technology can enhance the sport watching experience by collecting audience heart rate data in a stadium announcing the collective heart rate at key moments in the game such as scoring events through public displays [27]. Page and Moere examined how wearable basketball jersey displays projecting game-relevant information can improve experiences for coaches, referees, and audiences [24]. We learn from these projects that introducing technology to existing sports games can be beneficial, not just for the athletes, but can also enhance the experience of audiences. The authors note the importance of not interfering with the primary goal of the audience: in this case, watching a hockey game. This work contributes to our knowledge of interactive displays in sport settings; however, the sensed data is coming from the audience, not the sports activity, which is what we are interested in, as in public spaces there could be moments where there is no audience. 


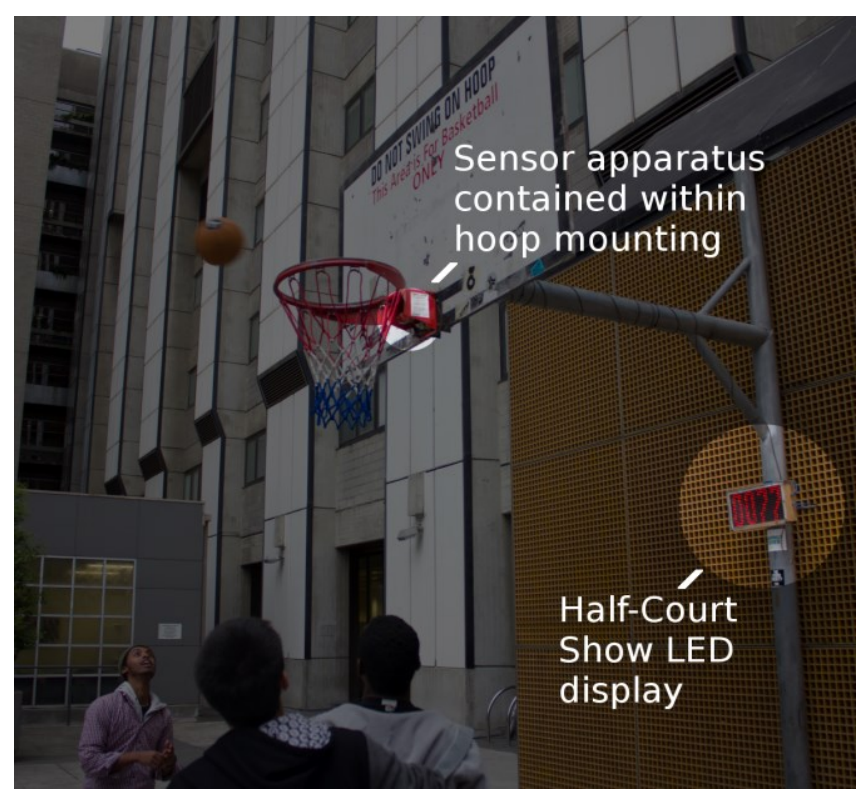

Figure 1: Half-Court Show

Another area from which we draw is research into the addition of digital display technology to existing exertion games. Ishii et al.'s Ping Pong Plus [11] is an example of using displayed visual information to enhance an existing game of ping pong. Ping Pong Plus demonstrated that such enhancement could increase player engagement and present players with an opportunity to create new ways of interaction on top of games they are familiar with. However, Ping Pong Plus exists as self-contained, standalone exhibit experience, whereas we are interested in how such enhancements could unfold in existing spaces where play is already taking place. Overall, what is missing is an understanding of such interactive displays in existing public game spaces.

Examining related work identifies a research gap: we lack knowledge about interactive displays in public game spaces; in particular, there is a limited understanding of how people use such a display, which could inform how such displays should be designed. In order to narrow this gap, we designed an interactive display and installed it on a half-court basketball hoop in an inner-city urban location and interviewed players, audience members, and passers-by about their experiences with it.

\section{HALF-COURT SHOW}

We call our system "Half-Court Show" (Figure 1). HalfCourt Show is installed on a half-court basketball court, with one hoop, located in a public urban space in a large metropolitan city (population 4 million). The court has no fence or similar demarcation around it, and is adjacent on one side to a park and on another side adjacent to a road that only allows pedestrians and cyclists. The basketball court is administered by our university. However, this is not obvious to passers-by, as there are no signs marking it university property. Although the majority of the people passing by and playing on the court appear to be affiliated with the university, there are also many non-university people using the space. People either pass by on their way to the university buildings nearby, or they use the road to reach their non-university destination in the city. We estimate that thousands of people pass this basketball court every day. There are usually people playing on the court between $10 \mathrm{am}$ and $11 \mathrm{pm}$, weather permitting. The layout of the space as well as its location makes it appear as a public space, and there is also no controlling who is using the space. Having the court administered by our university allowed us to gain permission to install our display easily, while our court is publicly accessible. Half-Court Show operated 24 hours a day for a period of 10 weeks. We did not provide any information about what the display was for or why it had been installed to the users of the space.

\section{Implementation}

Huang et al. suggest that the physical characteristics of urban displays are not necessarily correlated with user engagement [10]; nevertheless, we now provide details of our display in case others are inspired by ours and want to build their own.

Half Court Show displays information via an off-the-shelf $32 \times 16 \mathrm{~cm}$ red LED matrix display with a resolution of 1 LED per square centimeter (totaling 512 LEDs). We selected this LED matrix because it was readily available, could be easily controlled by custom-made electronics in real-time, and is very cost-effective. An infrared sensor is attached beneath the rim of the hoop to detect when a basket is made. The display is enclosed in our own wood and polycarbonate case and attached to the hoop pole. An Arduino microcontroller inside the case processes the sensor signal and generates the display data. We had to consider a number of special design requirements for HalfCourt Show:

- Durable: The design needed to withstand both inclement weather as well as impacts from play and potential vandalism.

- Theft-resistant: Theft is an issue in public spaces, so the design specifically avoided valuable components (for example, an LCD screen).

- Inexpensive: In addition to the risks above, the typical budgetary constraints of many public game facilities encouraged us to keep the display cost low. Our display cost less than $\$ 100$ in parts.

\section{Display Modes}

We decided to display 3 different modes over the course of our study. In creating these modes, we were inspired by our initial observations of public play, the related literature, and our combined 15 years of experience in designing technology for sports activities. Our 3 modes were: a cumulative basket counter, a basket timer, and a mode displaying motivational text and light insults - "trash talk". 


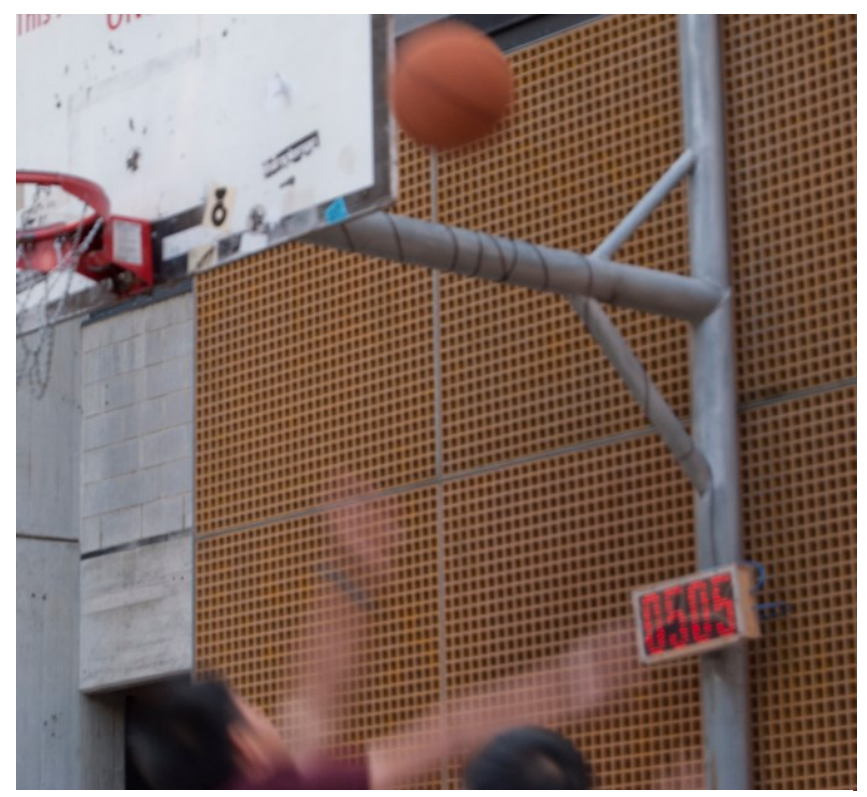

Figure 2: Cumulative Score Display

Cumulative Score Display

During the first four weeks, our display showed the number of baskets scored throughout the day (Figure 2), starting at 0 every morning. Over the course of the day, the number would increase as players shot baskets, with 700 baskets being a typical number displayed around sunset. For this mode, we were inspired by peripheral displays that make information visible that is otherwise not easily available, such as Mankoff et al.'s Daylight Display, a lamp which mirrors the sunrise and sunset to provide indoor users with knowledge of impending nighttime [15]. Although traditional score counters make the amount of baskets per team visible, they usually reset at the end of the game, whereas our cumulative basket counter counts any basket (regardless of shot by whom) and keeps counting throughout the day automatically, whether a game finished or not.

\section{Timer Display}

After the first 4 weeks, we changed Half-Court Show to display the time between baskets and examined its effects for another 3 weeks. After a basket was scored, the number of seconds since the last basket was flashed (meaning it turned on and off rapidly) for two seconds, and then the display showed an incrementing counter counting seconds from zero (Figure 3). After five minutes of no scoring, the display would begin scrolling text displaying the average time between baskets. This mode was inspired by player feedback and our observation of basketball being a rapid game in which time is very important: organized basketball features a shot clock which counts down a fixed number of seconds, and the attacking team has only that much time available to attempt a shot, motivating a fast paced game [21]. In contrast to a shot clock, our basket timer counts up, as we wanted to allow flexibility for users to come up with creative uses for this information.

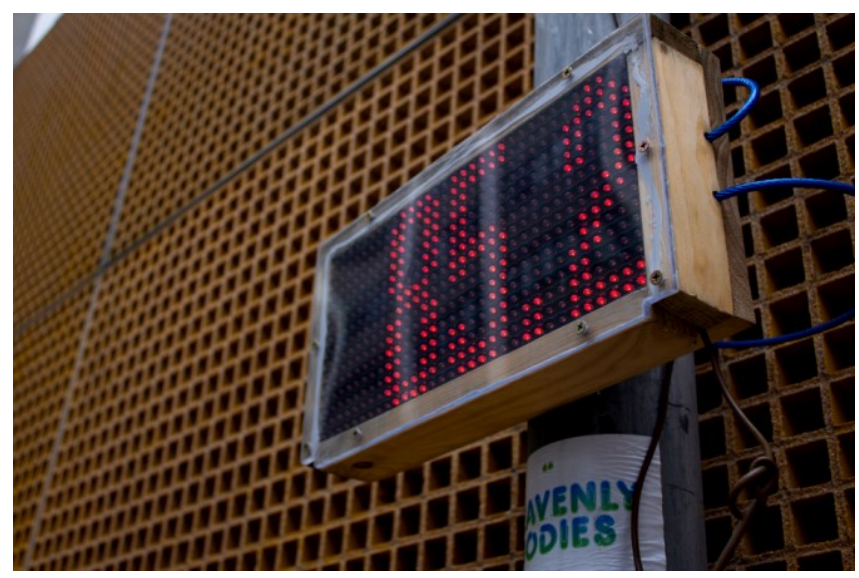

Figure 3: Timer Display

Text Display

During the final three weeks of the study, we implemented a third mode: the display flashes "!!!!!!" 3 times after a basket was made (Figure 4), and then displays a short preprogrammed "trash talk" message, which scrolls through if too long for the display length. The messages were a mix of positive and negative content, for example, "GREAT SHOT", "MARRY ME", "THAT WAS JUST LUCK", and "MY MOTHER SHOOTS BETTER". Our display selected these from a corpus of 311 phrases written by the research team and students and professors from a university writing class. The amount of messages and the selection algorithm (random, but only from previously not displayed messages) were chosen to minimize the chance for people seeing a message twice during play. The messages were scrolled across the display at a rate around 100 words per minute, as used by Kang and Muter [13]. Furthermore, when no baskets are made within a five minutes interval, the display would show a longer passage of text written by a professional comedian for the project. This text contained jokes and chants phrased as if coming from the basketball hoop if it could talk, for instance:

"MY FRIENDS ARE ALL ON PROTEIN SHAKES AND STUFF BUT I CAN'T SEE MYSELF DRINKING THEM

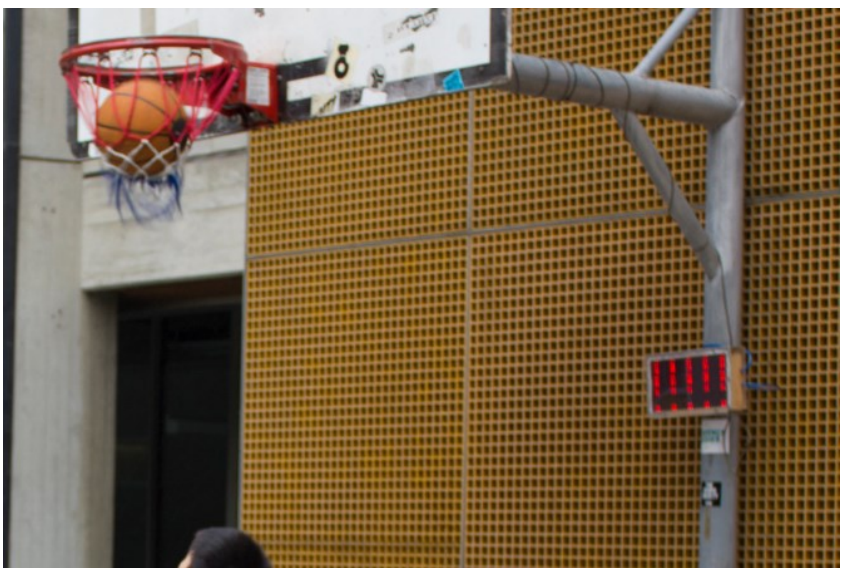

Figure 4: Text Display 
WELL MAINLY CAUSE I DON'T HAVE A DIGESTIVE SYSTEM".

This text display mode was inspired by the power of carefully crafted words, as celebrated in creative writing, and the use of trash talk in sports contexts, particularly in basketball, while also drawing from the use of chants and jokes in supporting sports activity. Unlike displays in stadiums that use motivational chants and jokes to entertain the crowd, controlled by a professional announcer, our mode is autonomous and draws from a preselected corpus, with the system knowing nothing about the activity besides that a basket was scored. Nevertheless, with this mode we intended to explore how direct motivation (in the form of encouraging statements and trash talk) could affect user experience, as well as how the standby text could affect perceptions of the space when no basketball was played.

\section{STUDY}

In our study, we conducted 164 semi-structured interviews in situ over 10 weeks. We interviewed 86 players and 78 non-players in the space. Our participants were chosen based on their physical presence in the area around the basketball court. We conducted interviews at different times of day, as well as on weekdays and weekends. We interviewed players, players waiting to play, spectators, and passers-by. We focused on questions relating to interactions with the display, always considering their specific actions in the space, for example we asked player how their gameplay changed in response to the display, and passersby how their passing-by changed in response to their play. We asked how the specific display mode changed their behavior, but also about people's perception of the space, the players, and the activity in the space. Interviews lasted usually between 2 and 15 minutes, depending on how much time participants were willing to give. We also asked participants to compare the current mode to the previous modes when they experienced one of the previous modes.

Interviews were audio and video recorded, then transcribed and analyzed using qualitative video analysis software (NVivo) to find important recurrent themes. We refined and grouped these findings using affinity diagrams in order to derive a set of key concepts, which we discussed amongst the researchers to construct our design understanding. Based on this, we articulated a set of design dimensions that characterize the design space of these displays as well as a set of strategies for the design of future interactive displays in public game spaces.

\section{Limitations}

It is important to note that our study was conducted "in the wild" [22], and such, was necessarily less controlled than a traditional user study. As a consequence, our users experienced the display modes in a specific order and may not have experienced all three. Additionally, our interviews had less consistency in terms of format and length than one would expect in a controlled experimental study. Furthermore, the recruitment of participants was based on the people in the space during times when the research team joined the space, and the data we collected represents only those people willing to take the time to talk to us. However, our sample includes participants from a wide range of ages and backgrounds, including construction workers, professors, and returning alumni. Previous research on public displays highlights the challenges of conducting such interviews in the wild [22], yet reported insightful results. Considering this, we believe our "in the wild" approach is particularly suited to the informal context of street basketball.

\section{EXPERIENCING HALF-COURT SHOW}

A public game space is used by a wide range of people, not just game players. We considered this with our interviews and therefore captured the experiences of a wide range of people. Inspired by Sheridan's categorization of participants exposed to an exertion game at a public festival [29], we group our interviewees into the following categories:

- Players: people present on the court playing or intending to play basketball.

- Inhabitants: people not involved in playing basketball. Inhabitants included people who actively spectated but never played, as well as people who were merely waiting around the area for other reasons, such as eating lunch or smoking or passing-by.

We use these categories to understand any potentially different reactions to the display as well as to examine if and how the display mediates connections between these groups that often occupy the same public space.

Our results, which we label as (F)indings, fall into three overarching categories: how the display is noticeable (Noticeability), how it supports play (Support for users), and how it facilitates a community of inhabitants of the space (Community).

\section{Noticeability}

Our first category of findings describes how participants noticed the display and directed their attention to it.

\section{(F1) Being aware of the display}

Out of the 164 people we interviewed, all 86 players noticed the display, but 38 of the 78 inhabitants had not become aware of it. This initially surprised us, as the display was located at eye level on the supporting pole of the hoop, displaying bright red LEDs, so that people watching the hoop would be facing it. However, numerous studies on inattention blindness show that even extreme events commonly remain unseen when people are focusing on watching something else, like people playing basketball [30]. In the rest of our findings, we will only refer to the remaining 126 interviewees, as we now focus on how people interacted with the display. 
(F2) Appreciating that users can control their level of engagement with the display

15 players and 1 inhabitant mentioned that they appreciated the way in which the display "faded" into the background while playing or watching the game. As the display was attached to the pole of the basketball hoop, outside of the main focus area of the game, it appeared it was relatively easy for people to be in control of their level of engagement with the content of the display:

Inhabitant 34 (Score): "I like how it's not in your face, and it's just there as assistance, for people to just enjoy their day"

Player 45 (Timer): “It's cool even if you didn't like it, you know, it's not big and in your face like every, like I know every time I shoot like I don't really focus on it, but now the words I just look at it every now and then to see what it says, it's cool"

Player 80 (Text): "You notice it when we're just like taking shots [...] but when you're playing you don't really pay much attention to it",

\section{(F3) Disrupting gameplay}

However, it was also mentioned that the display could have potential for being distracting, both due to engagement with the content and by the physical properties of the light, with 1 player and 1 inhabitant noticing some degree of disruption to gameplay.

Player 16 (Score): “...whenever we go to shoot, you just like see it, and you get distracted from it. Before, when it wasn't there, like a month ago, it was easier like, you got no red dots in your eyes"

Inhabitant 62 (Text): "You're displaying something that people stop and ok there's something coming and they stop and read and they have a laugh"

\section{Support for users}

Our next set of findings concerns how the display supported the players and inhabitants in the space.

(F4) Drawing motivation from display content

Players expressed that they drew motivation from the display content. Players were motivated to not only score more baskets, but also to play longer, and they felt encouraged to come back to play.

Player 15 (Score): "[I] try and hit shots to keep it ticking."

Player 20 (Score): "It makes you want to score more points."

Interviewer: "So you play longer?"

Player 20: "Yep. So you can get more points on."

Player 81 (Text): “...just seeing something there as like recognition from when you get your shots in, kinda keeps you going",
In addition to motivating exertion, players mentioned that the display provided emotional support and built confidence:

Player 61 (Text): “... a bit of a confidence booster; feeds the ego"

Player 77 (Text): "As a, um, amateur shooter, I can just, you know, have confidence about my shooting and stuff, feel good about myself",

Player 86 (Text): "[It] gets you pumped up"

(F5) Using the display to set goals

9 players said they used both the score and timer display to set goals:

Player 9 (Score): “...it can record our score, and like, today our target is 1000”,

Player 44 (Timer): “...if you shot it and you put it in in like 10 seconds, then [...] next time you're like trying to beat this, trying to get it faster, like 8 seconds, 7 seconds",

(F6) Trying to reach large round numbers

With the score display, 7 of our players specifically mentioned setting goals at large round numbers and showed excitement when such a number was reached.

Player 7 (Score) to Player 6: “...you got to 1000, it was like: damn!"

Player 23 (Score): “It was like 595, we're like, you know what, we'll get 600 and just quit playing"

(F7) Seeking recognition for performance

13 of our players and 1 inhabitant mentioned that they used the display as a way of confirming that a basket did indeed go in, even though it is usually quite clear when a basket is made:

Player 8 (Score): "When you score, you know you scored"

Player 61 (Text): "Yeah it's pretty cool, really cool, reassuring it's going in"

(F8) Unmet expectations of display content diminishing engagement

When the display was showing the timer mode, 13 participants explicitly compared the timer with an official basketball shot clock. In 7 of these cases, the mismatch between the expected action of a shot clock and the actual behavior of the display made the players consider the display to be irrelevant to them.

Player 41 (Timer): "Even like counting down, if you had... 'cause shot clocks are either 24 or 35 seconds. We're not playing serious stuff, so, if you had a 35 second countdown, we might actually use it then"

Inhabitant 60 (Timer): "...if I'd be playing in a normal match I'd be looking at towards the shot clock [...]. Here it's like the same thing but not really" 
(F9) Arising fantasy feelings due to the display

The display also elicited feelings of fantasy from 10 players. The display made players feel that they were in a more official setting, which they considered positive.

"Player 45 (Timer): You know that normal court, yeah like NBA or something, they have that scoreboard, sort of things like that, so you feel like on court, you're like some professional"

"Player 66 (Text): Sometimes I feel excited, like you shoot the ball then, it gives you a message. You, you can imagine that you are playing in the NBA, at the final shot-'yeah, I win!",

\section{Community}

Our final category of findings concerns how our display supported a community of inhabitants of the space.

(F10) Fueling social interaction between players

With the text mode, players would not only laugh together when funny quotes were displayed, but they would also read and point out quotes to others, both to share the experience as well as leveraging the comments to brag and trash talk with other players.

Player 79 (Text): "It's good, like, if you score on someone and get that, come on. A bit, rub it in"

Player 86 (Text): "You say exactly what you see on the thing [display] itself",

(F11) Increasing feelings of participation between the audience and the players

With the text display, the shared experience of reading the messages on the display with others made spectators watching the display feel more part of the overall experience.

Inhabitant 62 (Text): "As people sitting there and watching, all of us are even if, you feel very involved because all of us are laughing at the same thing"

Inhabitant 78 (Text): "Even though I don't play, just by watching it, and reading it we, sort of were participating anyway"

\section{(F12) Connecting people across time}

Finally, when the display showed the cumulative daily score, it drove awareness to the overall court usage over a longer period of time, even when no players were currently present. People in the space appreciated knowing that the space was used by others, and they used the score as a proxy for the amount of activity on the court. This shared awareness offered players and non-players an opportunity to connect across time with other users of the space.

Inhabitant 6 (Score): "And it really does add to the space I think, like not so much the device there, but like the playing there adds a lot to the space, um, and it just sort of illustrates that."
Player 11 (Score): "I guess they've been playing quite long cause sometimes I see like 1000 points right there, I'm like, impressive!"

Inhabitant 17 (Score): “...normally here I'm here like Saturdays [...] at least it makes me feel that I'm not the only one being around. Which can be motivating."

\section{DESIGN DIMENSIONS}

Through our findings as well as through our experience of designing Half-Court Show, we derived a set of design dimensions for interactive displays in public game spaces. These dimensions span a design space for these displays and help designers be aware of the various options that exist for them when creating future systems.

\section{Dimension 1: Balancing noticeability across different user groups}

Our first dimension describes the degree to which the display balances the noticeability across different user groups, and is therefore concerned with how the display attracts the attention of the different public game space users. The dimension of noticeability has been explored previously in analyses of peripheral displays, including the work of Matthews et al., as well as in Pousman and Stasko's dimension of notification levels [16][28]. However, these analyses focus on the interruption and visibility aspects concerned with a single user.

As our findings indicate, having multiple groups of users in a public game space interacting with the display can add complexity - a display that goes so far as to even interrupting players in a space (F3) can still go unnoticed by people actively watching a game (F1). Depending on how the designer intends to impact the experiences of different users, they must balance the needs of different groups - for instance, a bright, flashy display designed to aggressively grab the attention of spectators would likely get more engagement from passers-by, but would likely disrupt the players. Half-Court Show placed an emphasis on not disrupting the play already occurring in the space, and largely accomplished that goal (F3) while still being appreciated by players for the way it provided additional information (F2). However, the large number of inhabitants who remained completely unaware of the space (F1) shows us an opportunity to try and increase engagement with those users.

\section{Dimension 2: Support for different play actions}

Our second dimension describes how the display supports play action. Public games generally have multiple aspects to them that the designer may want to influence independently. Although a display in the sporting context is generally associated with elements of formal game rules, such as game time and team score, in a public game space, where informal play and practice occur alongside more structured play, there are many opportunities to support activities beyond standard rule sets. For instance, our score counter increased duration of play across multiple rounds of play. However, there will be tradeoffs when supporting 
different play actions. While our score counter encouraged longer play, it seemed to have little impact on intensity, while the timer mode caused some users to set up goals based on rapid-fire shooting, a higher intensity activity (F5). Finally, there may be elements outside the direct gameplay that the designer may want to influence, such as the social interactions between players, for example see those encouraged by our text display (F10).

\section{Dimension 3: Support for connecting user groups}

Our final dimension is concerned with the extent to which the display supports different user groups connecting. As such, the dimension depicts the ways in which the display can support the dynamics between groups of users. We unpack this further, examining how displays affect and facilitate any relationships between groups of simultaneous users and between groups using the display at different times.

Dimension 3.1: Support for concurrent shared experiences This characteristic of the dimension concerns how the display interacts with different user groups simultaneously to create shared experiences. Firstly, the display content can be tuned to be less or more relevant to social interaction. With Half-Court Show, most of the goal setting with the score and timer displays was aimed towards individual goals, while the text display encouraged trash-talk, an activity facilitating social interaction (F10). Furthermore, display content that draws the interest of different user groups can create a sense of shared experience (F11) [19], so conversely, display content tuned specifically towards one group of users may not interest others, thus possibly not generating the same sense of shared experience. Secondly, as McCarthy establishes [18], by controlling positioning and other physical design elements, the designer can influence expectations as to who the intended audience is. For instance, facing the display away from the players sends a signal that the display is intended for others. Therefore, in considering how to display data, designers can influence how the display might impact how concurrent users are connecting.

Dimension 3.2: Support for shared experiences across time In addition to facilitating experiences shared by groups of users concurrently using the display, the designer can alter the temporal window of the experience, connecting different users across time. In Half-Court Show, the timer and text displays only recorded the action of the most recent basket. In contrast, by extending the experience over a full day, the score display was able to communicate information between different user groups at different times, giving both players and inhabitants a feeling of the overall use of the space (F12). Different users of the space might have different access patterns over time (for instance, students walking through the space multiple times a day, or players who only come on Saturday mornings), therefore designers should consider the timing aspect of the information they aim to display to support different user groups connecting.

\section{DESIGN STRATEGIES}

We now articulate a set of 6 design strategies we derived from our work. While our above design dimensions span an abstract design space of interactive displays in public game spaces, the following strategies complement them by offering practical lessons for designers as to how to build better interactive displays for public game spaces. Each strategy relates to one of the categories identified: Noticeability, Support for users, and Community.

\section{Noticeability}

Strategy 1: Design for ignorability to not disrupt the play activity

In the same vein as Mankoff et al.'s heuristic for ambient displays [15] that designers should allow for letting the display "fade into the background", we also suggest that in public game spaces it is important that inhabitants are able to let the display fade into the background (F2). This is especially important given the potential to disrupt play activity (F3). We therefore recommend designers to be aware of any interruptions the display could provide to the user (D1), so that it can benefit some participants while respecting others who want to continue play in the space as they have so far without the display. To achieve this goal, we suggest designing towards greater "ignorability". For example, we recommend forgoing large screens - as Huang et al. point out [10], the size of a display is not necessarily related to the amount of interaction it drives, but a larger display may provide for a greater amount of distraction.

Strategy 2: Change display content when no play is
occurring Most exertion games feature some sort of break in the form of half-time, timeouts or handovers between goals. As we saw with our text display, even only displaying content during these breaks can support engagement while allowing players to ignore the display during the play activity (F2). Additionally, designers should consider how to drive engagement with the display during periods of no play at all. Without players to interrupt, the display can try to attract more attention, combatting the unawareness we experienced (F1).

\section{Support for users}

Strategy 3: Use context to drive feelings of fantasy

One of our most unexpected results was the sense of fantasy that our display was able to evoke in players. Research in computer games has previously highlighted the potential of interactive technology to support the fantasy element in players [14]. Here we extend this work and suggest that digital displays can facilitate the fantasy element not only in the indoor sedentary living room, but also in outdoor public game spaces, with players expressing delight in being supported in imagining playing in the NBA, for example (F9). By installing a display with similar characteristics as one in a professional stadium, players were able to partly imagine themselves as professional athletes, increasing their motivation (F9). Interestingly, this fantasy notion 
appeared with both display content very similar to a professional display (timer display) and one with little similarity (text display).

Strategy 4: Motivate play by showing new and unexpected display content

As the sense of fantasy elicited by our display seemed to be independent of the content displayed, our fourth design strategy is for designers to create new experiences rather than leaning on the form and function of score displays that currently exist within professional sporting venues. While our first two displays drew heavily from existing scoreboard concepts, our final text mode drove more excitement and greater engagement from both players and spectators. In contrast, both the score counter and timer mode, based off of existing score displays, seemed to prime users to want the real score or shot clock displays, and as a result, not to interact with the display to the same extent (F8).

\section{Strategy 5: Exploit the appeal of numbers to increase exertion investment}

Our players picked up the ball once more to get the display change from 999 to 1000 (F6). We know from gaming, and in particular gambling, that numbers can have a strong appeal to people and that the anticipation of number changes can be powerful drivers for action. Furthermore, presenting a number to people can "anchor" their expectations of what an average or reasonable target number should be [12], for example our players used the score display to set their own goals (F5). Our research demonstrates that the value of numbers matter to people in a public game space, and we therefore recommend that designers exploit the appeal of numbers and number changes to support the anticipation of reaching self-set goals.

\section{Community}

Strategy 6: Share data across time to facilitate inhabitants' belonging to the space

With the score display, the information we presented to the public was shared over the course of a 24-hour period. What surprised us was how this simple sharing provided both players and inhabitants with information about the court that allowed them to feel the presence of those in the space before them. For example, this feeling motivated one of our inhabitants, a researcher working in an adjacent building, to work harder because he knew he was not alone on campus during weekends (F12). In addition, this sense increased our users' appreciation for the play space, and contributed to them perceiving themselves as part of a greater community of people in the space (F12). Therefore, by simply presenting data across time, designers can similarly foster a greater sense of community within the space.

\section{CONCLUSION}

We presented Half-Court Show, an interactive display we designed for a public basketball half-court in order to study people's interactions with it. This extends prior work on displays in public non-game spaces by contributing the first systematic understanding of the interactions with a display in a public space designed for play. We found that interactive displays can support users of a public game space both by motivating players and facilitating a sense of community between inhabitants of the space. Designers of such displays need to consider a set of design dimensions specific to the context of public game spaces: balancing noticeability across different user groups, support for different play action, and support for connecting user groups. We also presented 6 strategies outlining practical recommendations for designers within the design space spanned by those dimensions in order to successfully support and connect users without distracting or disrupting their primary activities.

Our findings are limited by the specificity of our setup. Different sports and locations might impact the ways in which an interactive display is used, and implementing more systems across a wider variety of public game spaces could expand our understanding in this regard. Nevertheless, our results are the first important step towards this understanding of interactive displays in public game spaces and our research suggests the potential of such displays to make positive contributions.

Our work aims to help researchers and designers better understand the design space of interactive displays in public game spaces. As display technology becomes increasingly cheaper and widespread, we expect to see such displays appear in more public play spaces, from tennis courts to football fields to playgrounds. By offering guidance towards designing these displays, we hope to positively shape this future and inspire others to design displays that enhance public play spaces and support people in utilizing the play opportunities these spaces offer, ultimately supporting more urban play.

\section{ACKNOWLEDGMENTS}

We like to thank David Carlin and Simon Taylor for writing witty statements for the display, Eric Dittloff and Helmut Munz for video help, all our interviewees, and RMIT's property services for their help and encouragement. We would also like to thank Jen Mankoff, Sebastian Deterding, Wouter Walmink, Danielle Wilde, Sebastiaan Pijnappel, Ruth Sancho Huerga, Harry Lee, Chad Toprak, Gina Moore, Rohit Khot, and Joshua Platt for their helpful feedback. This research was performed under the Australian Research Council's DP110101304 grant.

\section{REFERENCES}

1. Ault, A., Krogmeier, J.V., Dunlop, S.R., Coyle, E.J., "eStadium: The Mobile Wireless Football Experience". In Proc. ICIW 2008, 644-649.

2. Bentley, F. R. and Groble, M. TuVista: meeting the multimedia needs of mobile sports fans. In Proc.MM 2009. ACM Press (2009), New York, NY, 471-480. 
3. Brignull, H., and Rogers, Y. Enticing People to Interact with Large Public Displays in Public Spaces. In Proc. INTERACT 2003, ACM Press (2003).

4. Figl, K. and Gerhardt, T., Motivational and training effects of technical cycling equipment. In Proc. IADIS, (2008), 365-367.

5. Foth, M., Schroeter, R., and Ti, J. Opportunities of public transport experience enhancements with mobile services and urban screens. International Journal of Ambient Computing and Intelligence (2013), 1-18.

6. Gobster, P. Managing Urban Parks for a Racially and Ethnically Diverse Clientele. Leisure Sciences, 24-2, Taylor \& Frances (2002), 143-159.

7. http://www.fedsquare.com/events/

8. http://www.gesturetek.com/gesturefx/productsolutions groundfx.php

9. http://nikeplus.nike.com/plus/products/gps_app/

10.Huang, E.M., Koster, A., and Borchers, J. Overcoming Assumptions and Uncovering Practices: When Does the Public Really Look at Public Displays? In Pervasive Computing 2008, Springer (2008), 228-243.

11. Ishii, H., Wisneski, C., Orbanes, J., Chun, B., and Paradiso, J. PingPongPlus: design of an athletic-tangible interface for computer-supported cooperative play. In Proc. CHI 1999, ACM Press (1999), 394-401.

12. Kahneman, Daniel. Thinking, Fast and Slow. Farrar, Straus and Giroux, New York (2011).

13. Kang, T. J. I. N., and Muter, P. Behaviour \& Information Technology Reading dynamically displayed text. Behaviour \& Information Technology, 8(1)(1989), 33-42.

14.Lazzaro, N. 4 Fun Keys: Testing Emotions and Player Experiences. In Game Usability: Advice from the Experts for Advancing the Player Experience, Morgan Kaufmann, 2008

15. Mankoff, J., Dey, A. K., Hsieh, G., Kientz, J., Lederer, S., and Ames, M. Heuristic evaluation of ambient displays. In Proc. CHI 2003, ACM Press (2003), 169176.

16. Matthews, T., Dey, A. K., Mankoff, J., Carter, S., and Rattenbury, T. A toolkit for managing user attention in peripheral displays. In Proc. UIST 2004, ACM Press (2004), 247-256.

17. Matthews, T., Rattenbury, T., and Carter, S. (2007). Defining, Designing, and Evaluating Peripheral Displays: An Analysis Using Activity Theory. HumanComputer Interaction, 22, 1-2 (2007), 221-261.

18. McCarthy, A. Ambient Television: Visual Culture and Public Space. Duke University Press, Durham, NC (2001).
19. Meyrowitz, J. No Sense of Place: The Impact of Electronic Media on Social Behavior. Oxford University Press (1985).

20. Mueller, F., Edge, D., Vetere, F., Gibbs, M. R., Agamanolis, S., Bongers, B., and Sheridan, J. G. Designing sports: a framework for exertion games. In Proc. CHI 2011, ACM Press (2011), 2651-2660.

21. National Basketball Association. History of the Shot Clock. http://www.nba.com/analysis/00422949.html

22. O'Hara, K, and Glancy, M. Understanding collective play in an urban screen game. In Proc. CSCW 2008, ACM Press (2008), 67-76.

23. O'Hara, Kenton, Perry, M., and Lewis, S. Social Coordination around a situated display appliance. In Proc. CHI 2003, ACM Press (2003), 66-72.

24.Page, M., and Moere, A. V. Evaluating a wearable display jersey for augmenting team sports awareness. In Pervasive Computing, 4480, 1-18.

25.Paulos, E. and Jenkins, T. Urban probes: encountering our emerging urban atmospheres. In Proc. CHI 2005, ACM Press (2005), 341-350.

26. Peltonen, P., Kurvinen, E., Salovaara, A., Jacucci, G., Ilmonen, T., Evans, J., Oulasvirta, A., and Saarikko. P. It's Mine, Don't Touch!: interactions at a large multitouch display in a city centre. In Proc. CHI 2008, ACM Press (2008), 1285-1294.

27.Perttula, A., Tuomi, P., Suominen, M., Koivisto, A., and Multisilta, J. (2010). Users as sensors: creating shared experiences in co-creational spaces by collective heart rate. In Proc. 14th International Academic MindTrek Conference, ACM Press (2010), 41-48.

28. Pousman, Z., and Stasko, J. A taxonomy of ambient information systems. In Proc. AVI 2006, ACM Press (2006), 67-74.

29. Sheridan, J. and Bryan-Kinns, N. Designing for Performative Tangible Interaction. International Journal of Arts and Technology. Special Issue on Tangible and Embedded Interaction, 1-3/4, Inderscience Publishers (2008), 288-308

30. Simons, D. J., and Chabris, C. F. (1999). Gorillas in our midst: Sustained inattentional blindness for dynamic events. Perception-London, 28(9), 1059-1074.

31. Sturm, J., Herwijnen, O. H., Eyck, A., and Terken, J. Influencing social dynamics in meetings through a peripheral display. In Proc. ICMI 2007, ACM Press (2007), 263-270.

32. Toprak, C., Platt, J., Ho, H., and Mueller, F. Cart-LoadO-Fun: Designing Digital Games for Trams. In $\mathrm{CHI}$ 2013 EA, ACM Press (2013), 2877-2878. 